\title{
"Réveiller la Vénus endormie » : le plaisir sexuel et ses limites dans le discours médical de la première moitié $\mathrm{du} \mathrm{XV}^{\mathrm{e}}$ siècle
}

Estela Bonnaffoux

\section{CpenEdition}

\section{Journals}

Édition électronique

URL : http://journals.openedition.org/questes/4444

DOI : 10.4000/questes.4444

ISSN : 2109-9472

\section{Éditeur}

Les Amis de Questes

\section{Édition imprimée}

Date de publication : 31 janvier 2018

Pagination : $51-68$

ISSN : 2102-7188

\section{Référence électronique}

Estela Bonnaffoux, « «Réveiller la Vénus endormie » : le plaisir sexuel et ses limites dans le discours médical de la première moitié du Xve siècle», Questes [En ligne], 37 | 2018, mis en ligne le 01 février 2018, consulté le 30 avril 2019. URL : http://journals.openedition.org/questes/4444 ; DOI : 10.4000/ questes.4444 


\title{
« Réveiller la Vénus endormie » : le plaisir sexuel et ses limites dans le discours médical de

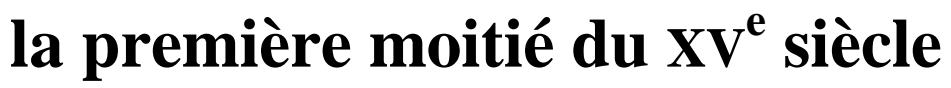

\author{
Estela BONNAFFOUX \\ Centre d'Études Supérieures de la Renaissance, Tours
}

Écrire sur le plaisir sexuel? Une telle idée peut paraitre incompatible avec la théologie chrétienne. Toutefois, les enjeux liés à la génération ont très tôt permis un discours médical sur ce sujet, dont les concepts fondamentaux trouvent leur origine dans les écrits d'Hippocrate, d'Aristote et de Galien. Dans le cadre de la physiologie hippocraticogalénique, la prise en compte du plaisir, notamment féminin, est ainsi indispensable à la procréation. En effet, la semence émise par la femme lorsqu'elle éprouve du plaisir a un pouvoir fécondant. Les ouvrages d'Aristote la présentent davantage comme un réceptacle de la semence de l'homme: son but serait de fournir la matière tandis que le sperme masculin seul détiendrait la vertu formative susceptible d'imprimer la vie à cette matière. Cependant, même si l'absence de plaisir de la femme ne met pas en péril la conception, Aristote avance que, lorsque le coït s'accompagne d'une mutuelle jouissance, le passage du sperme masculin s'en trouve facilité. La médecine médiévale, héritière du débat sur l'existence ou non d'un sperme féminin, a bien souvent cherché à concilier ces deux positions opposées. La question de la nécessité d'une 
émission des deux partenaires est en tout cas cruciale, en ce qu'elle pose les bases d'un lien entre plaisir et conception ${ }^{1}$.

Dans la pensée scientifique de la première moitié $\mathrm{du} \mathrm{Xv}^{\mathrm{e}}$ siècle, ces différentes théories sous-tendent toujours les discours sur la génération et sont enrichies par l'apport de la culture arabe, dont les œuvres majeures, comme le Canon d'Avicenne, sont étudiées à l'université. Nourrie de cette influence, la médecine médiévale s'est érigée, selon les termes de Danielle Jacquart et de Claude Thomasset, en véritable « $\operatorname{art}^{\text {érotique }}{ }^{2}$ ». En renforçant l'idée selon laquelle le plaisir (et particulièrement le plaisir féminin) est indispensable à la procréation, le Canon d'Avicenne permet de contourner l'interdit de l'amour charnel : au sein du couple marié, le plaisir devient parfaitement légitime ${ }^{3}$. Bien plus, les médecins présentent l'absence de plaisir comme une des causes d'union inféconde. C'est donc fréquemment dans une optique de guérison de la stérilité que se développe un discours médical sur le plaisir sexuel, comme l'illustrent les trois auteurs de notre étude, tous issus de l'université de Padoue : Bartolomeo

\footnotetext{
${ }^{1}$ Sur la réception médiévale des théories de la génération, consulter Maaike Van der Lugt, Le Ver, le démon et la vierge. Les théories médiévales de la génération extraordinaire: une étude sur les rapports entre théologie, philosophie naturelle et médecine, Paris, Les Belles Lettres, 2004.

${ }^{2}$ Danielle Jacquart et Claude Thomasset, Sexualité et savoir médical au Moyen Âge, Paris, PUF, 1985, p. 121-192. Voir aussi Jean-Claude Bologne, La Naissance interdite : stérilité, a vortement, contraception au Moyen Âge, Paris, Orban, 1988, p. 246-260.

${ }^{3}$ Sur la sexualité au Moyen Âge, consulter Michel Foucault, Histoire de la sexualité. I. La volonté de savoir, Paris, Gallimard, 1976. Sur les liens entre sexualité et médecine, voir Jean Verdon, Le Plaisir au Moyen Âge [1996], Paris, Perrin, 2010 et Amour, sexualité et médecine aux $\mathrm{Xv}^{\mathrm{e}}$ et $\mathrm{XvI}^{\mathrm{e}}$ siècles, dir. Olga Anna Duhl, Dijon, Éditions universitaires de Dijon, 2009. Pour une époque ultérieure à notre propos, voir Robert Muchembled, L'Orgasme et l'Occident. Une histoire du plaisir du Xvi siècle à nos jours, Paris, Seuil, 2005, notamment "Chapitre 1. Ce plaisir qu'on dit charnel...», p. 27-68. Pour une étude des pratiques et mœurs sexuelles chez Girolamo Mercuriale (1530-1606), consulter Jean-Michel Agasse, «Désir, plaisir et pratiques sexuelles sous le regard d'un médecin de la Renaissance », Seizième siècle, vol. 7, n 1, 2011, p. 85-97.
} 
Montagnana ${ }^{4}$ et Michel Savonarole ${ }^{5}$ intègrent l'acte sexuel à leurs chapitres respectifs sur la stérilité ${ }^{6}$ et sur les moyens de concevoir ${ }^{7}$, tandis qu'Antonio Guaineri $^{8}$ consacre un chapitre entier à l'art de « réveiller la Vénus endormie ${ }^{9} »$ au sein de son ouvrage sur les maladies de la matrice.

Condamné dans d'autres sources, le plaisir sexuel trouve une justification médicale; mais parce que l'érotisme touche à l'interdit, le discours des médecins se teinte de prudence. En se fondant sur l'autorité d'Avicenne, ceux-ci décrivent alors un rapport sexuel normé, qui oscille entre tabous et transgressions. Enfin, si la femme peine à se dégager du double poids de la morale chrétienne et de la théorie aristotélicienne, qui font d'elle un être faible, intrinsèquement pécheur, et résolument passif,

${ }^{4}$ Bartolomeo Montagnana (ca. 1380-ca. 1452) exerça à l'université de Padoue entre
1422 et 1441. Il est l'auteur de nombreux consilia médicaux, écrits entre 1428 et 1448.
Sur Bartolomeo Montagnana, voir Jole Agrimi et Chiara Crisciani, Les "Consilia »
médicaux, trad. Caroline Viola, Turnhout, Brepols, 1994. Consulter également le
Dizionario Biografico degli Italiani en ligne, sur Treccani : http://www.treccani.it/enciclopedia/bartolomeo-montagnana_(Dizionario-Biografico)/ (page consultée le 21/04/2016).

${ }^{5}$ Formé à Padoue puis professeur à l'université de Ferrare, Michel Savonarole (ca. 1384-1468) entra au service du marquis d'Este à partir de 1440. Parue en 1479, l'ensemble de son œuvre fut réunie sous le titre Practica Maior. Sur Michel Savonarole, voir Michele Savonarola: medicina e cultura di corte, dir. Chiara Crisciani et Gabriella Zuccolin, Florence, SISMEL/Edizioni del Galuzzo, 2011.

${ }^{6}$ Bartolomeo Montagnana, Consilia, [1499], Consilium 206, De Sterilitate, Lyon, Jacques Myt, 1525, fol. 288ra.

${ }^{7}$ Michel Savonarole, Practica Maior, [1479], VI, 21, 23 De Ingeniis et aliis ad impregnationem facientibus, Venise, héritiers de Lucantonio Giunta, 1547, fol. 272ra.

${ }^{8}$ Né à Chieri, Antonio Guaineri (ca. 1390-ca. 1458) effectua une partie de ses études à l'université de Padoue, avant de rejoindre Pavie. Il enseigna dans cette même université à partir de 1412, avant de devenir médecin personnel du Duc de Savoie, Amédée VIII. Ses Opera Medica furent publiés en 1481 à Pavie. Sur Antonio Guaineri et la culture de cour, voir Marilyn Nicoud, Le Prince et les médecins. Pensée et pratiques médicales à Milan (1402-1476), Rome, École Française de Rome, 2014, notamment p. 46-47 et p. 170-174. Consulter également l'article «Antonio Guaineri » rédigé par Daniela Mugnai Carrara, dans le Dizionario Biografico degli Italiani en ligne, sur Treccani : http://www.treccani.it/enciclopedia/antonioguaineri_(Dizionario-Biografico)/ (page consultée le 21/04/2016).

${ }^{9}$ Antonio Guaineri, De Matricibus, XXIV De approximandis ab extra quae in coitu praestant delectationem et quae a proprietate Venerem sopitam excitant, Padoue, Conradus de Paderborn, 1474, fol. d2rb. 
son accès à un plaisir revalorisé rend possible une relative libération du corps féminin.

\section{La caution médicale}

Nécessaire à l'homme d'un point de vue social, puisqu'elle perpétue l'espèce, au $\mathrm{XV}^{\mathrm{e}}$ siècle la sexualité est également jugée indispensable au bon fonctionnement du corps. Au même titre que l'alimentation ou le sommeil, elle est en effet comprise dans les res non naturales, dont l'équilibre garantit une bonne santé. Pour être bénéfique et efficace, elle doit obéir à un principe de modération et être accomplie selon un ensemble de préceptes bien établis ${ }^{10}$. Le contrôle médical sur la sexualité semble exclure la recherche effrénée du plaisir seul. Éprouvée par l'homme et la femme, la «delectatio» est subordonnée à la procréation, dont elle n'est, comme l'explique Antonio Guaineri, qu'un « iuvamentum »:

Le plaisir que, dans l'acte du coït, prennent l'homme comme la femme, aide grandement, ou plutôt considérablement, la conception. C'est pourquoi je décrirai dans ce présent chapitre quelques délices du coït, et certaines choses propres à réveiller la Vénus endormie ${ }^{11}$.

\footnotetext{
${ }^{10}$ La médecine médiévale distingue en effet les choses naturelles (l'ensemble des composants physiologiques du corps), les choses non-naturelles (l'ensemble des paramètres extérieurs au corps, qui influent sur sa nature physiologique) et les choses contre nature (les maladies). Sur les choses non-naturelles, voir Luis Garcia Ballester, «On the origins of the Six Non-Natural Things in Galen», Galen und das hellenistische Erbe, dir. Jutta Kollesch et Diethard Nickel, Stuttgart, Steiner, 1993, p. 105-115; Pedro Gil Sotres, «Les régimes de santé », Histoire de la pensée médicale en Occident, dir. Mirko Dražen Grmek, Paris, Seuil, 1995, t. I, p. 257-281 et Marilyn Nicoud, Les Régimes de santé au Moyen Âge : naissance et diffusion d'une écriture médicale, $\mathrm{XIII}-\mathrm{XV}$ siècles, plus particulièrement t. I, chap. IV, «Autour des choses non-naturelles », p. 153-184, Rome, École Française de Rome, 2007.

11 Antonio Guaineri, De Matricibus, op. cit., XIX, fol.d2rb : «Delectatio in actu coitus, quam vir similiter et mulier capiunt magnum, immo maximum, ad conceptionem iuvamentum praestat. Ea de re, nonnulla coitum delectantia et aliqua a
} 
Présenté comme un moyen de lutter contre la stérilité, le plaisir est en réalité un élément quasiment indispensable pour procréer : sans être clairement exprimée, cette idée est fortement induite par la construction même de la phrase, qui corrige l'adjectif «magnum » par son superlatif « maximum ». Bartolomeo Montagnana arrive à une conclusion similaire lorsqu'il explique que les «tristes inquiétudes de l'âme [...] empêchent grandement la génération» («Tristes animi sollicitudines [...] ingenter generationem impediunt $\left.{ }^{12} »\right)$, et qu'au contraire le plaisir la parachève («generatio enim delectatione perficitur $\left.{ }^{13} »\right)$. Réaffirmer le lien entre plaisir et conception autorise les développements sur les «délices du coït» et vise à légitimer un discours médical qui s'inscrit ici dans la lignée du discours religieux.

Les médecins abordent en effet un sujet qui, sans être nouveau, demeure difficile, dans une société où le sexe revêt un but strictement procréateur. Toute évocation des pratiques sexuelles et du plaisir qui en découle, puisqu'elle va à l'encontre de la morale chrétienne, devient dès lors suspecte et nécessite une constante justification. Tout en revendiquant un statut de praticiens qui les autorise à délivrer des conseils sans équivoques, les médecins ont conscience des réactions de honte et de pudeur attachées à la sexualité : toujours présent, le souci de ne pas heurter la bienséance les engage à montrer une certaine retenue. Ils n'ont alors de cesse de prouver "l'honnêteté » de leurs propos, une notion récurrente chez nos trois auteurs et que Katharine Park définit comme le «fondement de la vertu féminine et de l'honneur masculin dans les

proprietate sopitam Venerem excitantia [...] in praesenti capitulo describam ». Sauf indication contraire, nous traduisons.

${ }^{12}$ Bartolomeo Montagnana, op. cit., De Sterilitate, Consilium 207, fol. 289va.

${ }^{13}$ Ibid. 
familles patriciennes ${ }^{14} »$. Bien qu'il expose «les actes nécessaires à la fécondation», Bartolomeo Montagnana assure qu'il le fera «en préservant la moralité et l'honnêteté ${ }^{15}$ ». Michel Savonarole indique qu'il « [n'hésitera] pas à écrire les choses utiles à la génération, même si elles ne semblent pas honnêtes à entendre ${ }^{16} »$, avant d'ajouter : «Je laisse à la prudence du médecin ce que la plume ne peut écrire ${ }^{17} \gg$. Tous ces exemples illustrent bien la position délicate du médecin, dont la liberté de ton se heurte constamment au tabou sexuel. Révélateur de l'opinion courante qui tend à célébrer la virginité féminine, le proverbe populaire rapporté par Antonio Guaineri, «Ce qu'on dit chez les gens : "une femme honnête meurt sans enfant", le savant ne le dit jamais ${ }^{18}{ }$, est aussi l'occasion de rappeler que, loin de porter un regard réprobateur sur la sexualité, l'homme de science tient en ce domaine son propre discours, qui n'appartient pas aux gens du commun et qui tend à se démarquer des préceptes religieux.

Sans rejeter le plaisir, les médecins cherchent à l'intégrer aux préceptes de santé et à le réglementer; toute la difficulté consiste à concilier franc-parler médical et respect des bienséances. Issus de l'université, ils partagent un héritage commun qu'ils restituent dans leurs traités. En matière de sexualité, ils s'appuient sur Galien et ses disciples islamiques, et tout particulièrement sur le Canon d'Avicenne, qui offre la

\footnotetext{
${ }^{14}$ Katharine Park, Secrets de femmes : le genre, la génération et les origines de la dissection humaine, Dijon, Les Presses du Réel, 2009, p. 122.

${ }^{15}$ Bartolomeo Montagnana, De Sterilitate, op. cit., Consilium 206, fol. 288vb : « Actus necessarios ad fecundationem, salva semper reverentia moralitatis et honestatis ».

${ }^{16}$ Michel Savonarole, De Ingeniis et aliis ad impregnationem facientibus, op. cit., VI, 21, 23, fol. 272rb : «Non dubitabo scribere quae sunt utilia generationi, etsi non videantur auditui honesta ».

${ }^{17}$ Ibid., «Prudentiae medici relinquo quod calamo scribi non potest ».

${ }^{18}$ Antonio Guaineri, De Matricibus, op. cit., XIX, fol.c5vb : «In vulgari aiunt: "honesta mulier sine prole moritur", sapiens numquam ».
} 
description détaillée d'un acte sexuel strictement hétérosexuel ${ }^{19}$. Un passage en particulier, dans lequel Avicenne conseille de «prolonger le jeu » avec la femme, apparaît dans les traités des trois médecins évoqués :

Que l'homme prolonge le jeu avec les femmes dont la complexion n'est pas mauvaise. Qu'il touche donc les seins de la femme, qu'il touche son pubis, en se gardant de s'unir à elle par une véritable étreinte. Et lorsqu'il le désire et le veut, qu'il s'unisse à elle, tout en lui frottant de haut en bas l'espace entre l'anus et la vulve. C'est là le lieu de son plaisir. Qu'il soit bien attentif au moment où elle renforce son étreinte, où ses yeux commencent à rougir, où elle se met à haleter et à balbutier: qu'il émette alors son sperme au fond du col de l'utérus ${ }^{20}$.

Maintes fois repris, le passage en question permet de déléguer la parole et d'évoquer l'acte sexuel à travers une description convenue, émanant d'une autorité médicale parfaitement reconnue. La portée médicale de tels propos, sous lesquels pourrait affleurer un érotisme condamnable, se trouve alors réaffirmée.

La reprise systématique de certains éléments invite à se demander si ce discours figé, qui a presque valeur topique, n'impose pas finalement une nouvelle norme dont les médecins peineraient à s'affranchir. Nous

\footnotetext{
${ }^{19}$ Bartolomeo Montagnana condamne ainsi les mœurs sodomites, qui sont pour lui de véritables «abominations » au même titre que la bestialité, De Sterilitate, op. cit., Consilium 15, fol. 38vb.

${ }^{20}$ Avicenne, Canon medicinae [1473], trad. Gérard de Crémone, Venise, Dyonisius Bertochus, 1490, liber III, fen XXI, tract. I, cap. X, fol. t2ra : "Tangat ergo vir eius mamillas cum facilitate et tangat pectinem eius, et obviet ei non permiscendo se ei permixtione vera. Cumque desiderat et affectat, permisceatur ei fricando de ea, quod est inter anum eius desuper et vulvam. Ille enim locus est locus delectationis eius. Consideret ergo in ea horam in qua fortis fit in ipsa adherentia, et incipiunt oculi eius mutari in rubedinem, et eius anhelitus elevari et verba eius balbutire, et tunc mittat illic sperma oppositum ori matrici ». La traduction d'Andrea Alpago propose une variante intéressante (Avicennae liber canonis, [1527] trad. Andrea Alpago, Venise, héritiers de Lucantonio Giunta, 1555, fol. 383v) : "permisceatur ei fricando de ea, quod est inter labia eius desuper » («qu'il s'unisse à elle, en lui frottant de bas en haut l'espace entre les lèvres »).
} 
verrons cependant qu'au-delà des ressemblances, les trois exemples étudiés laissent entrevoir la part de liberté et d'auto-censure propre à chaque médecin.

\section{Dire et ne pas dire l'acte sexuel}

Le Canon d'Avicenne décrit un rapport sexuel s'effectuant en deux temps. Il s'agit tout d'abord de préparer la femme au coït Avicenne énumère les préliminaires, qu'il nomme «ludus », et qui constituent de fait des actes ne se limitant pas à la simple pénétration. D’une manière générale, Michel Savonarole suit ces conseils, tout en y ajoutant des détails inspirés de Constantin 1'Africain ${ }^{21}$. L'homme allie les gestes aux paroles, les caresses aux baisers :

L'homme doit toucher la femme tout autour des seins, avec douceur, et surtout l'extrémité des seins; y joindre des baisers, dire des paroles provocantes, et dans le même temps approcher sa verge de la vulve, sans l'y introduire, toucher enfin, et en quelque sorte frotter, l'endroit qui se trouve entre la vulve et l'anus : c'est le lieu d'un immense plaisir ${ }^{22}$.

Antonio Guaineri réalise une synthèse similaire à celle de Michel Savonarole :

Avant donc d'en venir au coït, l'homme se place familièrement dans les bras de la femme, et tout en lui donnant des baisers d'une suave succion des lèvres, il dit des mots doux et qui inspirent

\footnotetext{
${ }^{21}$ Constantin l'Africain, Liber de coitu, II, 16, cité par Enrique Monterro Cartelle et Maria Cruz Herrero Ingelmo, «Conceptión y erotismo en la literatura médica medieval », Cuadernos de Filología Clásica. Estudios Latinos, vol. 32, n 2, 2012, p. 303.

${ }^{22}$ Michel Savonarole, De Ingeniis et aliis ad impregnationem facientibus, op. cit., VI, 21, 23, fol.272rb : "Debet vir mulierem tangere ut circa mamillas et leuiter et specialiter capita mamillarum, oscula iungere, et verba provocantia dicere, et cum virga vulvae appropinquando non intromittendo, et ultimo tangendo et aliqualiter fricando locum medium inter vulvam et anum et est locus maxime delectationis ».
} 
l'amour, il palpe doucement des doigts les tétons des seins, et parce que dans un tel acte il faut tout faire, il frotte doucement l'endroit entre la vulve et l'anus. En effet, cette friction, comme le rapportent plusieurs auteurs, procure à la femme un immense plaisir ${ }^{23}$.

Les deux auteurs expriment la douceur et l'intimité d'un amour conjugal qui prône certes l'initiative masculine, mais accorde une grande place à l'exploration du corps féminin, longuement étreint, caressé et embrassé. Tous ces éléments contrastent fortement avec la description donnée par Bartolomeo Montagnana, incluse dans une série de préceptes contre la stérilité :

Que l'homme prête aussi attention à ce chapitre, parce qu'il ne va pas de soi qu'avant la copulation, il lui faut, pendant environ une heure, jouer avec la femme, et inversement, avec des baisers, en touchant les seins, les mamelons et les parties génitales, en occupant ainsi ce temps jusqu'à ce que l'homme voie la femme passer de pâle à rouge, que sa respiration devienne un court instant plus fréquente, et qu'il sente sous ses doigts un léger soubresaut autour des parties du bas-ventre et des mamelles. C'est alors que l'homme s'unira à la femme $[\ldots]^{24}$.

Seul à sous-entendre une participation active de la femme dans les ébats («cum muliere et econverso »), le médecin atténue toutefois

\footnotetext{
${ }^{23}$ Antonio Guaineri, De Matricibus, op. cit., XXIV, fol. d2va : «Antequam igitur ad coitum, vir accedat in mulieris amplexibus familiariter se ponat, et cum suavi labiorum succione oscula det, plurima verba dulcia et amorem inducentia proferat mammillarum papillas digitis leviter pertractet, et quia in actu tali omnia facere fas est locum inter anum et vulvam leviter confricet. Ea enim fricatio, ut auctores ferunt, plurimi in muliere delectationem affert maximam ».

${ }^{24}$ Bartolomeo Montagnana, De Sterilitate, op. cit., Consilium 206, fol. 288vb : «Et audiat etiam vir capitulum istud. Itaque parum videlicet per unam horam aut circa ante copulationem ludatur cum muliere et econverso, cum osculis et tactu mamillarum et suminis et partium genitalium, tempus hoc expendendo quousque vir viderit mulierem discoloratam factam rubicundam et anhelitum parumper frequentatum et levem motum saltuosum circa partes inguinales et suminis perceptum sensu tactus. Tunc ergo vir copulet se mulieri ».
} 
quelque peu l'érotisme, à travers la mention de zones érogènes réduites à l'essentiel (seins, mamelons, parties génitales). Bien plus, Bartolomeo Montagnana élude le moment de la pénétration, préférant aux discours plus détaillés de ses collègues une expression que l'on pourrait presque qualifier de litote (« en occupant ce temps »). Jamais nommé, le plaisir se fait moins explicite: il est à chercher dans les diverses manifestations physiques agitant le corps féminin, dont certaines sont déjà décrites dans le Canon avicennien.

Selon Avicenne, en effet, l'homme doit scruter le visage de la femme pour y déceler le plaisir sexuel, qui annonce l'émission de la semence féminine ; alors seulement peut-il procéder à la pénétration et à l'éjaculation. Si Michel Savonarole ${ }^{25}$ reste fidèle aux critères établis par Avicenne (les yeux de la femme rougissent, sa respiration se précipite et elle se met à balbutier), Bartolomeo Montagnana et Antonio Guaineri adaptent légèrement ce discours. Le premier conserve la rougeur de la femme, sans l'appliquer aux yeux, tandis que des spasmes trahissent son plaisir. Les yeux ne « rougissent $^{26} »$ plus chez Guaineri, ils « scintillent $^{27} »$, et la femme, gagnée par le trouble, devient incapable de s'exprimer :

Lorsque les yeux de la femme scintillent, et qu'elle dit des mots tronqués, et qu'elle s'arrête,

\footnotetext{
${ }^{25}$ Michel Savonarole, De Ingeniis et aliis ad impregnationem facientibus, op. cit., VI, 21, 23, fol. 272rb : «Oculi eius rubeant et anhelitus elevetur et quod cum lingua balbutiet $\gg$.

${ }^{26}$ La traduction de «rubeant» et de «rubicundam» demeure problématique : une analyse du texte arabe permettrait de déterminer si ces deux termes expriment un éclat particulièrement vif des yeux féminins. La traduction d'Andrea Alpago conserve l'idée de rougeur adoptée par Gérard de Crémone (Avicennae liber canonis, op. cit., fol. $383 \mathrm{v})$.

${ }^{27}$ Antonio Guaineri, De Matricibus, op. cit., XXIV, fol. d2va : «Saepe ut venereum actum perficiat, operam det donec tamen mulieris oculos scintillare percipiat» ( Souvent, pour parfaire l'acte de Vénus, l'homme doit s'y appliquer fréquemment ; jusqu'à ce que toutefois il perçoive les yeux de la femme scintiller »).
} 
fatiguée, au milieu de sa phrase, et que dans ses mouvements elle n'a aucune mesure, alors qu'aussitôt l'homme mastique du poivre ou du cubèbe, et qu'il s'enduise la verge de la salive causée par une telle mastication, ou qu'il fasse de même, avec du fiel de chèvre et de la poudre de graines d'ortie, ou de la poudre de satyrion, ou qu'il fabrique une sorte d'onguent avec du miel qu'il applique sur sa verge. Ou bien fais ainsi: prends des parts égales de pyrèthre d'Afrique, de staphisaigre, de cannelle, broie l'ensemble avec minutie, et filtre-les avec une étoffe de soie, puis avec du sirop, après avoir confit du gingembre avec du sirop fais des pilules, garde l'une d'elles dans la bouche, puis avec la salive qui en est produite, enduis la verge et le col de la matrice ${ }^{28}$.

Une fois suscité, le plaisir doit être prolongé tout au long de l'acte sexuel. Par conséquent, aux caresses succède l'utilisation de produits réputés pour leurs vertus aphrodisiaques, et que les médecins consignent dans leurs traités. Michel Savonarole conseille ainsi d'enduire l'entrée de la vulve avec de l'huile de lys blanc ${ }^{29}$. Les recettes données prennent ici une dimension parfaitement concrète : appliqués directement ou ingérés sous forme de pilule, ces aphrodisiaques ont une double fonction. Tout en excitant le désir, ils se mêlent à la salive pour former un lubrifiant à appliquer sur les parties génitales. Leur emploi se justifie en ce qu'ils

\footnotetext{
${ }^{28}$ Ibid. : «Cumque mulieris scintillant oculi ac verba truncata loquitur, sic quod in medio suorum verborum lassata sistit, et in motibus suis modum habet nullum, tunc vir statim piper aut cubebas masticet, et cum saliva ex tali masticatione causata virgam illiniat, vel idem cum felle capre et pulvere seminis urticae faciat, vel pulverem satirionis in modum ungenti cum melle conficiat cum quo tunc virgam inungat. Vel facias sic accipe piretri, stafisagriae, cinamomi, ana partes equales, terantur subtilissime et cum cindato cribrentur deinde cum sirupo conditi zinzibri pillulas confice, ex quibus unam in ore retine et cum saliva inde genita virgam ac matricis collum illiniat ».

${ }^{29}$ Michel Savonarole, De Ingeniis et aliis ad impregnationem facientibus, op. cit., VI, VI, 21, 23, fol. 273va.
} 
participent du succès de la relation sexuelle et favorisent par là même la génération.

Révélatrices des choix que les médecins opèrent, les variations observées dans chaque passage montrent que se conformer au cadre établi par Avicenne n'interdit pas pour autant de faire preuve d'originalité. Les praticiens s'autorisent ajouts et développement, voire se livrent, à l'image de Bartolomeo Montagnana, à une atténuation de l'érotisme, qui tend, sinon à taire entièrement le plaisir, du moins à le rendre plus implicite.

\section{Vers une revalorisation du plaisir ?}

Bien que très codifiées, ces descriptions de l'acte sexuel contiennent en elles les prémices d'une revalorisation des plaisirs masculin et féminin. Cette idée est particulièrement nette chez Antonio Guaineri et Michel Savonarole, qui évoquent le plaisir des deux partenaires, dont les émissions respectives sont nécessaires pour que s'ensuive la conception. Antonio Guaineri écrit ainsi qu' «après avoir diligemment observé ce qui a été dit, mari et femme s'acquitteront vigoureusement de leur dette envers dame Vénus, en prenant soin d'émettre ensemble leur semence au même instant ${ }^{30} »$. Michel Savonarole précise lui aussi que, pour concevoir, l'homme et la femme «doivent émettre [leur semence] en même temps ${ }^{31}$ », et que « dans le coït même, [la femme] doit éprouver un plaisir outre nature ${ }^{32} »$, utilisant pour cette dernière phrase une tournure pléonastique (delectetur...delectationem) qui

\footnotetext{
${ }^{30}$ Antonio Guaineri, De Matricibus, op. cit., XXIV, fol. d2va : «Dominae Veneri debitum finale persolvant obnixe studendo, ut in eadem instanti spermata simul emittant ».

${ }^{31}$ Michel Savonarole, De Ingeniis et aliis ad impregnationem facientibus, op. cit., VI, 21, 24, fol. 273va : « Ita requiritur ut eodem tempore emittant ».

${ }^{32}$ Ibid., VI, 21, 23, fol. 272ra: «In coitu ipso delectetur ultra naturalem delectationem ».
} 
met l'accent sur la delectatio féminine. Antonio Guaineri emploie encore un verbe explicite, «spermatizet », qui laisse peu de doute concernant le rôle de la femme dans la génération : il explique qu'appliquée sur la verge, l'huile de castoréum provoque chez la femme «un tel plaisir qu'elle produit tout de suite de la semence ${ }^{33} »$. Au même titre que l'homme, la femme émet une semence (spermatizet), à condition toutefois qu'elle éprouve du plaisir. Accorder de l'importance à ce fait revient à prendre en compte le corps féminin.

Tous ces débats se trouvent clairement atténués chez Bartolomeo Montagnana. S'il concède que le plaisir « parachève la conception », il se garde de préciser qui l'éprouve et le bannit complètement de la description qu'il donne du coït, alors qu'il indique volontiers par ailleurs que la matrice reçoit du plaisir ${ }^{34}$. Le médecin ne se détache manifestement pas de l'image de la femme réceptacle dans laquelle, dit-il, l'homme répand sa semence («effuso in ipsa semine $\left.{ }^{35} »\right)$. Cet exemple tend à prouver que l'évocation du plaisir féminin se heurte malgré tout aux représentations traditionnelles associées à la femme, et dont les médecins gardent l'empreinte dans leurs écrits, tout en relayant la théorie d'une part active de la femme dans le processus de génération.

Une expression bien particulière permet toutefois de superposer, sans contradiction, diverses images attachées à la femme en matière de

\footnotetext{
${ }^{33}$ Antonio Guaineri, De Matricibus, op. cit., fol. $\mathrm{d} 2 \mathrm{vb}:$ : Si tu in priapis oculo de castoreo oleum imposueris tantam enim ex hoc mulier delectationem accipit ut citissime spermatizet $\gg$.

34 Bartolomeo Montagnana, De Sterilitate, op. cit., Consilium 208, fol. 272ra : « Neque delectationem convenientem ex velocitate exitus eius accipit matrix» («la matrice ne retire pas un plaisir convenable d'une éjaculation trop rapide »).

35 Ibid., Consilium 207, fol. 289va : «Cum senserit anhelitum frequentatum magis quam prius, tunc, effuso in ipsa semine, parumper stet ei copulatus » («lorsque [l'homme] sentira que la respiration de la femme est plus fréquente qu'auparavant, alors, après avoir répandu sa semence en elle, qu'il reste un instant uni à elle »).
} 
sexualité : la «Vénus endormie ${ }^{36} »$, que l'on trouve dans le De matricibus d'Antonio Guaineri. Dans la littérature médicale, la femme est résolument pudique $^{37}$ et ne détient pas l'initiative dans le domaine de la sexualité. En adéquation avec la morale chrétienne, l'idée d'une femme soumise à l'homme durant l'acte sexuel est illustrée par la promotion de l'unique position sexuelle acceptable: «l'homme est sur la femme, et non l'inverse ». Dans cette position optimale (que la femme doit maintenir après le coït pour augmenter ses chances de concevoir), l'utérus conserve le sperme sans que «la semence féminine ne retombe dans la verge ${ }^{38}$ ». Placée sous l'homme, la femme se tient tête baissée («demisso capite $^{39} »$ ), les hanches et les jambes relevées le plus possible ( $«$ anchis elevatis ${ }^{40} »$, «cruribus ad alta notabiliter elevatis $\left.{ }^{41} »\right)$, tandis que l'homme enlace le dos de sa compagne et lui tient fermement les fesses («dorsum mulieri applicet quantum potest trahendo natices $\left.{ }^{42} »\right)$. Pour Antonio Guaineri, elle doit replier son pied gauche sous sa hanche («sinistrum pedem sub ancha reponat»), et maintenir l'autre tendu

\footnotetext{
${ }^{36}$ Antonio Guaineri, De Matricibus, op. cit., XXIV, fol. d2rb : « Sopita Venus ».

${ }^{37}$ Voir par exemple le lien entre pudeur féminine et sommeil chez Arnaud de Villeneuve (Hec sunt opera Arnaldi de Villanova que in hoc volumine continentur, [1475], Balthasaris de Babiano, Lyon, 1504, De sterilitate, fol. 253ra) : «Vir autem, quia mulieres sunt verecunde, dum dormierint primum somnum debet mulieris palpare mammillas et ventrem et excitare et ad coitum provocare » («Et parce que les femmes sont pudiques, l'homme doit, lorsqu'elles dorment d'un sommeil léger, palper leur poitrine et leur ventre, et les exciter et les inciter au coït »). Sur la pudeur, consulter Jean-Claude Bologne, Histoire de la pudeur [1986], Paris, Hachette, 1997, et plus récemment le premier numéro de la revue Histoire, médecine et santé, vol. 1, Pudeurs, Toulouse, Méridiennes, 2012.

${ }^{38}$ Michel Savonarole, De Ingeniis et aliis ad impregnationem facientibus, op. cit., VI, 21, 23, fol. 257rb : «Mas super feminam stet, et non econtra, ne ex spermate mulieris in virga aliquid cadat $\gg$.

${ }^{39}$ Antonio Guaineri, De Matricibus, op. cit., XXIV, fol. d2va.

${ }^{40}$ Ibid.

${ }^{41}$ Bartolomeo Montagnana, De Sterilitate, op. cit., Consilium 206, fol. 288vb.

${ }^{42}$ Ibid.
} 
(« dextrum autem extensum teneat $\left.{ }^{43} »\right)$. Il y a là une forme de soumission qui se trouve mimée jusque dans le symbolisme des pieds : le gauche, traditionnellement associé au féminin, est replié ; tandis que le droit, qui caractérise le masculin, reste étendu ${ }^{44}$. La figure de la «Vénus endormie » permet de traduire la passivité sexuelle de la femme; toutefois, elle pourrait être la réminiscence d'une tradition plus ancienne, comme le montre une occurrence dans la Collectio Salernitana. Employée pour désigner la baisse ou l'absence du désir féminin, l'expression «sopita venus » figure parmi les critères d'une grossesse imminente, au même titre que l'arrêt du flux menstruel ou le gonflement des seins ${ }^{45}$. Lorsqu'il parle du désir plus lent de certaines femmes, Antonio Guaineri utilise l'adjectif « sopitus » et indique comment « réveiller » et « vivifier chez de telles femmes leur appétit sexuel endormi ${ }^{46} »$. L’idée selon laquelle « la femme d'ordinaire tarde plus que $l^{\prime}$ homme ${ }^{47} »$ est admise par les médecins: la Vénus endormie qu'il faut réveiller devient donc une incarnation de la libido féminine, que l'homme doit solliciter avec adresse et patience. Les médecins l'enjoignent ainsi à attendre le plaisir parfois plus lent de sa partenaire, sans songer à satisfaire uniquement le sien. Dès l'Antiquité, ce thème de la Vénus endormie dans un paysage printanier et champêtre est en outre caractéristique des épithalames: ces poèmes offerts lors des noces mettent fréquemment en scène la déesse réveillée

\footnotetext{
${ }^{43}$ Antonio Guaineri, De Matricibus, op. cit., fol. d2va.

${ }^{44}$ Sur cette correspondance entre côté gauche et féminin d'une part, et côté droit et masculin d'autre part, voir par exemple Hippocrate, Aphorismes, V, 38.

45 Collectio Salernitana, Charles Daremberg et Salvatore de Renzi, Naples, Typographie du Filiatre-Sebezo, 1859, Cap. XXII, Signa conceptionis, p. 65.

${ }^{46}$ Antonio Guaineri, De Matricibus, op. cit., fol. d2vb : «Nonnullae quae parum aut minimum coitum appetunt, ut igitur in talibus sopitum coitus appetitum vivifices ».

${ }^{47}$ Michel Savonarole, De Ingeniis et aliis ad impregnationem facientibus, op. cit., VI, 21, 23, fol. 273va: "Quia mulier consuevit plus tardare quam vir, ideo debet se retinere vir, ut potest, ab emissione ».
} 
par Cupidon ${ }^{48}$. Elle se hâte alors de rejoindre le mariage qu'elle doit présider et adresse ses vœux aux époux, qu'elle bénit pour leur procurer un mariage fécond. Cette tradition se perpétue en Italie, puisqu'il était d'usage $a u \mathrm{XV}^{\mathrm{e}}$ siècle d'offrir des tableaux destinés à être accrochés dans la chambre conjugale. Ceux-ci se caractérisent par la représentation d'un nu féminin allongé, invitation à consommer le mariage ${ }^{49}$. C'est peut-être toutes ces images qu'Antonio Guaineri cherche à faire émerger chez ses contemporains : une Vénus qui, sous l'apparence d'un sommeil passif, offre implicitement le spectacle sensuel de l'amour physique. Elle se pare donc des caractéristiques négatives que l'on attribue volontiers à son sexe : la promotion du plaisir féminin ne s'effectue qu'à travers le prisme de la femme inactive et assujettie à l'homme. Les médecins peuvent ainsi célébrer la chair sans transgresser entièrement l'interdit.

La Vénus endormie cristallise plusieurs images de la femme. Elle incarne parfaitement le consensus dont ont pu faire preuve les médecins, entre évocation d'une liberté érotique et respect des normes sexuelles. Si cette image de la Vénus endormie sacrifie au topos de la femme soumise et passive, elle demeure une incitation au plaisir. La sopita Venus est certes la femme passive, dont la vue est éminemment érotique pour l'homme mais elle ne doit pas rester confinée dans ce rôle sous peine d'empêcher la conception : il est au contraire nécessaire de «réveiller » son plaisir, afin qu'elle puisse être pleinement Vénus.

48 Voir par exemple Claudien, «Epithalame de Pallade et Célérine », Euvres complètes, trad. Héguin de Guerle, Paris, Garnier Frères, 1865, p. 425.

${ }^{49}$ Sur le motif de la Vénus endormie, voir Keith Christiansen, « Lorenzo Lotto and the tradition of epithalamic painting », Apollo, vol. 124, 1986, p. 166-173 et Rona Goffen, «Renaissance Dreams », Renaissance Quarterly, vol. 40, n 4, 1987, p. 682-706, Daniel Arasse, «La femme dans le coffre », On n'y voit rien. Descriptions, Paris, Denoël, 2000, p. 107-152 et Virginie Leroux, "L'érotisme de la belle endormie », Seizième Siècle, n $^{\circ}$ 7, 2011, p. 15-35. 
En apparence exclu de la société médiévale, le plaisir sexuel fit en réalité l'objet de nombreuses réflexions parmi les médecins, qui y virent une aide indispensable à la perpétuation de l'espèce. Conformément au modèle avicennien, la relation sexuelle ainsi médicalisée est abordée à travers le cadre rassurant et légitime du couple hétérosexuel, moyen de braver l'interdit tout en évitant les condamnations. Pour qu'il y ait conception, il faut un désir mutuel ${ }^{50}$ : l'ambivalence d'un discours à caractère sexuel n'échappe pas à Bartolomeo Montagnana, qui préfère borner son discours à l'essentiel plutôt qu'encourir le risque de paraître «malhonnête ». Chez Antonio Guaineri et Michel Savonarole, le ludus de l'amour est amplement développé et les gestes érotiques, sans se limiter aux conseils médicaux, sont révélateurs d'une véritable tendresse entre époux. À travers l'attention portée à la douceur des étreintes et des baisers échangés, les médecins, bien que davantage guidés par la philosophie naturelle qu'animés par un réel intérêt vis-à-vis de la condition féminine, s'élèvent ainsi contre la vision réductrice qui fait de la femme un réceptacle passif. L'évocation poétique de la sopita Venus par Antonio Guaineri dessine l'image d'une femme dont le plaisir importe, même s'il est au service du but procréatif, et révèle en creux une nouvelle représentation de la femme, à la fois passive et sensuelle. Mais si le $\mathrm{XV}^{\mathrm{e}}$ siècle poursuit la libération des corps amorcée deux siècles auparavant, l'avènement de la langue vernaculaire pourrait bien avoir mis fin à «l'art érotique » des médecins. Désormais accessibles à tous, les écrits sur la génération renoncent à un plaisir sexuel redevenu indicible,

\footnotetext{
${ }^{50}$ Dans le cas contraire, l'union sera inféconde, comme le précise Michel Savonarole, qui fait figurer le défaut d'inclination dans les causes de stérilité (De Ingeniis et aliis ad impregnationem facientibus, op. cit., VI, 21, 24, fol. 273va) : «Errores autem impedientes utrosque sunt : primo ut cum vir et mulier non sunt cum amore copulati » («Voici les erreurs empêchant [la conception] chez les deux sexes : premièrement, lorsque l'homme et la femme ne sont pas unis par l'amour »).
} 
sous peine d'être soupçonnés, comme ce fut le cas pour Ambroise Paré, $\mathrm{d}^{\prime}$ « inciter la jeunesse à luxure ${ }^{51} »$.

${ }^{51}$ Voir à ce sujet Evelyne Berriot-Salvadore, Un Corps, un destin. La femme dans la médecine de la Renaissance, Paris/Genève, Champion/Slatkine, 1993, p. 77 et Dominique Brancher, «Les ambiguïtés de la pudeur dans le discours médical (15701620) », Cahiers de l'Association Internationale des études françaises, vol. 55, $\mathrm{n}^{\circ} 1$, 2003, p. 275-297. 